\title{
ON ANALYTIC SLIT MAPPINGS IN THE CLASS $\Sigma$
}

\author{
YUSUF ABU-MUHANNA AND Y. J. LEUNG
}

\begin{abstract}
We show that any analytic slit mapping is a support point in $\Sigma$. As an application, we use Schiffer's theory to show that a rational slit mapping must be of the form $z+a_{0}+a_{1} /\left(z-z_{1}\right)$.
\end{abstract}

I. Introduction. Let $\Sigma$ be the class of univalent functions analytic in $\tilde{\mathbf{D}}=$ $\{z:|z|>1\}$ with the expansion $f(z)=z+\sum_{n=0}^{\infty}\left(a_{n} / z^{n}\right)$ at infinity. The Koebe function $\kappa(z)=z+a_{0}+1 / z$ and its rotations are well-known examples in this class. They map $\tilde{\mathbf{D}}$ onto the complement of a line segment in the complex plane. Let $H(D)$ denote the complex linear space of analytic functions in a plane domain $D$ equipped with the usual topology of locally uniform convergence. A function $f$ in $\Sigma$ is called a support point if there is a continuous linear functional $L$ on $H(\tilde{\mathbf{D}})$ such that $\operatorname{Re} L(f) \geq \operatorname{Re} L(g)$ for all $g$ in $\Sigma$. Here we assume our functionals $L$ to satisfy $L(1)=0$ and nonconstant on $\Sigma$.

A function $K(z)=\sum_{n=1}^{\infty} \lambda_{n} z^{n}$ analytic in the closed unit disk $|z| \leq 1$ can represent a continuous linear functional $L$ on $H(\tilde{\mathbf{D}})$ in the form

$$
L(g)=\frac{1}{2 \pi} \int_{|z|=r} K(z) g(z) d z,
$$

where the circle $|z|=r>1$ lies inside the region of analyticity of $K(z)$. The omitted set $\Gamma$ of a support point $f$ lies on the trajectories of the quadratic differential $L(1 /(f-w)) d w^{2} . L(1 /(f-w))$ is an analytic function of $w$ in a set containing $\Gamma$. We refer the readers to Schober's lecture notes [12] or Duren's book [3] for proofs of these facts.

Suppose the quadratic differential $L(1 /(f-w)) d w^{2}$ has a zero of order $k$ at a point $w_{0}$ on $\Gamma$; several arcs up to the number $k+2$ can emanate from that point. By a second variation method, Chang et al. [2] showed that the angle between two arcs forking at a zero has to be at least $2 \pi / 5$. It is conjectured by Schober [13] that the zeros on $\Gamma$ are simple. For certain choices of $\lambda$ and $\mu$, the omitted sets of support points for $L(f)=a_{2}+\lambda a_{1}, a_{3}+\mu a_{1}$, respectively, have one and two simple zeros of $L(1 /(f-w))$. For other choices of $\lambda$ and $\mu$, the support points omit a single analytic arc lying on a noncritical trajectory of $L(1 /(f-w)) d w^{2}[8]$.

In contrast to the situation when the omitted set could fork, we show in this elementary note that any function in $\Sigma$ omitting a single analytic arc is a support point. Hence there are no restrictions on the geometry of $\Gamma$ in the present case except for the analyticity.

II. THEOREM. Let $f$ be in $\Sigma$. Suppose that the image of $f$ omits a single analytic arc. Then $f$ is a support point.

Received by the editors February 25, 1985.

1980 Mathematics Subject Classification. Primary 30C75.

(C) 1987 American Mathematical Society $0002-9939 / 87 \$ 1.00+\$ .25$ per page 
PROOF. Let $f(z)=z+\sum_{n=0}^{\infty} a_{n} / z^{n}$. Define $K(z)=\sum_{n=1}^{\infty} n \bar{a}_{n} z^{n-1}$. Since $f(z)$ is analytic on the unit circle, $K(z)$ is analytic in the closed unit disk $|z| \leq 1$. The functional $L(g)=\sum_{n-1}^{\infty} n \bar{a}_{n} b_{n}$ where $g(z)=\sum_{-\infty}^{\infty} b_{n} z^{-n}$ is continuous on $H(\tilde{\mathbf{D}})[\mathbf{1 2}$, Chapter 4].

For $g$ in $\Sigma$,

$$
\begin{aligned}
|L(g)| & =\left|\sum_{n=1}^{\infty} n \bar{a}_{n} b_{n}\right| \\
& \leq\left\{\sum_{n=1}^{\infty} n\left|a_{n}\right|^{2}\right\}^{1 / 2}\left\{\sum_{n=1}^{\infty} n\left|b_{n}\right|^{2}\right\}^{1 / 2} \leq 1=L(f) .
\end{aligned}
$$

Equality holds if and only if $g=f$. Thus we have shown that $f(z)$ is the unique support point for $L$.

The functional $L$ defined in the proof has the form

$$
L(g)=\frac{1}{2 \pi} \int_{|z|=r} K(z) g(z) d z
$$

where $K(z)=\left(1 / z^{2}\right)\left(1-\bar{f}^{\prime}(1 / z)\right) . \quad|z| \leq r$ is the closed disk in which $K(z)$ is analytic.

REMARKS. 1. From the theorem, we see that the omitted arc of an analytic slit mapping can be elongated analytically and then renormalized to produce a new support point in $\Sigma$. In the familiar class $S$ of univalent functions $f(z)=$ $z+\sum_{n=2}^{\infty} a_{n} z^{n}$ analytic in the disk $|z|<1$, the elongation process may not be able to produce new support points in $S[4]$.

2. Certain analytic slit mappings can support two different functionals. As mentioned earlier, for certain $\lambda$ 's, the support point for $L(f)=a_{2}+\lambda a_{1}$ omits a single arc. By the theorem above, it supports the related functional defined by $K(z)$. Except for the Koebe functions $z /\left(1+e^{i \theta} z\right)^{2}$, it is an open question in the class $S$ to produce functions that support at least two different functionals [4].

3. A function $f$ in $\Sigma$ is called an extreme point if $f$ cannot be written as $\lambda g+(1-\lambda) h$ with $0<\lambda<1$ and $g-h$ being nonconstant. $f$ is an extreme point if and only if it omits zero area (see $[5,3]$ ). The closure of the set of extreme points (in the locally uniform convergence topology of $H(\tilde{\mathbf{D}})$ ) is equal to the closure of the set of support points in $\Sigma$. So any function in $\Sigma$ that omits zero area can be approximated by a sequence of support points. This shows that the theorem above is anticipated.

III. In the last part of this note, we show that a rational function in $\Sigma$ omitting a single arc must be of the form $z+a_{0}+a /\left(z-z_{1}\right)$. This was proved recently by Uri Srebro [14] and St. Ruscheweyh [11]. Kühnau [7] pointed out that this problem was considered by L. Fuchs in the late 1800 s and subsequently by L. Bieberbach. At the risk of being called redundant, we shall give it another proof. We feel our proof illustrates the usefulness of Schiffer's approach to extremal problems.

PROPOSITION 1. If $f \in \Sigma$ is a rational slit mapping, then it omits either a straight line or a circular arc.

PROOF. We shall show that $f(z)$ has the form $z+a_{0}+a_{1} /\left(z-z_{1}\right)$. By the theorem above, the omitted arc $\Gamma$ of $f$ satisfies a Schiffer differential equation 
$L(1 /(f-w)) d w^{2}>0$. With $w=f(z)$, we have

$$
\left[\frac{1}{2 \pi} \int_{|\varsigma|=r} \frac{K(\varsigma)}{f(\varsigma)-f(z)} d \varsigma\right] z^{2} f^{\prime}(z)^{2}=Q(z)
$$

This is essentially Theorem 7.8 in Pommerenke's book [10]. Assume

$$
f(z)=z+a_{0}+\sum_{j=1}^{n_{0}} \frac{a_{j}}{z^{j}}+\sum_{\mu=1}^{m} \sum_{j=1}^{n_{\mu}} \frac{a_{\mu j}}{\left(z-z_{\mu}\right)^{j}} .
$$

$K(z)=\left(1 / z^{2}\right)\left(1-\bar{f}^{\prime}(1 / z)\right)$ has the form

$$
\sum_{j=1}^{n_{0}} j \bar{a}_{j} z^{j-1}+\sum_{\mu=1}^{m} \sum_{j=1}^{n_{\mu}} \frac{\lambda_{\mu j}}{\left(z-1 / \bar{z}_{\mu}\right)^{j+1}} .
$$

Hence $L$ is a functional of finite degree [10, p. 184]. $Q(z)$ is a rational function in $z$, nonpositive on the unit circle. We can follow Pfluger's argument (cf. [9]) in a similar fashion to show that $Q(z) / z^{2} f^{\prime}(z)=K(z)+$ analytic function in $|z|>1$.

At $\infty, Q(z)$ has a pole two degrees higher than $K(z)$. Otherwise, the location and the order of poles of $Q$ agree with those of $K(z)$ in $|z|>1$. On the right-hand side of (1), if $a_{n_{0}} \neq 0, Q(z)$ has a pole of order $n_{0}+1$ at 0 and $\infty$, poles of order $n_{\mu}+1$ at $z_{\mu}$ and $1 / \bar{z}_{\mu}$ for $\mu=1,2, \ldots, m$. The computation of the left side of (1) follows from the proof of Theorem 7.4 in [10]. We have

$$
P(w)=\frac{1}{2 \pi} \int_{|\zeta|=r} \frac{K(\zeta)}{f(\varsigma)-w} d \zeta=\sum_{j=1}^{n_{0}} \alpha_{j} w^{j-1}+\sum_{\mu=1}^{m} \sum_{j=1}^{n_{\mu}} \frac{\alpha_{\mu j}}{\left(f\left(1 / \bar{z}_{\mu}\right)-w\right)^{j+1}} .
$$

With $w=f(z),(1)$ becomes

$$
\left[\sum_{j=1}^{n_{0}} \alpha_{j} f(z)^{j-1}+\sum_{\mu=1}^{m} \sum_{j=1}^{n_{\mu}} \frac{\alpha_{\mu j}}{\left(f\left(1 / \bar{z}_{\mu}\right)-f(z)\right)^{j+1}}\right] z^{2} f^{\prime}(z)^{2}=Q(z) .
$$

Since $f(z)$ is rational, the equation above holds for all $z$. We shall prove the proposition by comparing the orders of poles on both sides of (2).

We push $z$ to $z_{1}$, say, then

$$
f(z)=O\left(1 /\left(z-z_{1}\right)^{n_{1}}\right) \text { and } f^{\prime}(z)^{2}=O\left(1 /\left(z-z_{1}\right)^{2 n_{1}+2}\right) .
$$

The left side of $(2)$ is $O\left(1 /\left(z-z_{1}\right)^{n_{1} n_{0}-n_{1}+2 n_{1}+2}\right)$ if $\alpha_{n_{0}}=n_{0} \bar{a}_{n_{0}} \neq 0$, while the right side behaves like $O\left(1 /\left(z-z_{1}\right)^{n_{1}+1}\right)$. Thus $n_{0} n_{1}+n_{1}+2=n_{1}+1$. This is impossible. So either (i) $f(z)$ has no poles outside the origin or, (ii) $\alpha_{n_{0}}=0$, which implies that $f(z)$ has no poles at the origin. In the first case, $f(z)=z+$ $a_{0}+\sum_{j=1}^{n_{0}} a_{i} / z^{j}$. We remark that this is Aharonov's case [1]. (2) becomes

$$
\left[\sum_{j=1}^{n_{0}} \alpha_{j} f(z)^{j-1}\right] z^{2} f^{\prime}(z)^{2}=Q(z)
$$

We push $z \rightarrow 0$ this time. The left side of (3) behaves like $O\left(1 / z^{n_{0}\left(n_{0}-1\right)+2 n_{0}}\right)$, while $Q(z)=O\left(1 / z^{n_{0}+1}\right)$. So we conclude that $n_{0}=1$. Hence $f(z)=z+a_{0}+a_{1} / z$. 
In the second case, (2) has the form

$$
\left[\sum_{\mu=1}^{m} \sum_{j=1}^{n_{\mu}} \frac{\alpha_{\mu j}}{\left(f\left(1 / \bar{z}_{\mu}\right)-f(z)\right)^{j+1}}\right] z^{2} f^{\prime}(z)^{2}=Q(z) .
$$

$Q(z)$ has poles at $z_{\mu}$ and $1 / \bar{z}_{\mu}$ both of order $n_{\mu}+1$ for $\mu=1, \ldots, m$. Around $z_{1}$ say, $f\left(1 / \bar{z}_{\mu}\right)-f(z)=O\left(z-z_{1}\right)^{-n_{1}}$. Hence the quantity inside the square brackets on the left side of $\left(3^{\prime}\right)$ behaves like $O\left(z-z_{1}\right)^{(k+1) n_{1}}$ for some positive integer $k$. Altogether, the left side has a pole of order $-(k+1) n_{1}+2 n_{1}+2$ at $z_{1}$ while the right side is of order $n_{1}+1$ there.

The equation $-(k+1) n_{1}+2 n_{1}+2=n_{1}+1$ yields $n_{1}=1$. This shows that all the poles of $f(z)$ are of the first order. We rewrite $\left(3^{\prime}\right)$ in the form

$$
\left[\sum_{\mu=1}^{m} \frac{\alpha_{\mu}}{\left(f\left(1 / \bar{z}_{\mu}\right)-f(z)\right)^{2}}\right] z^{2} f^{\prime}(z)^{2}=Q(z) .
$$

By comparing the order of growth of both sides around $z_{1}$ say, we conclude that $\sum_{\mu=1}^{m} \alpha_{\mu} \neq 0$.

Now push $z \rightarrow \infty$. Since $f(z) \sim z$ there, we have $Q(\infty)=\sum_{\mu=1}^{m} \alpha_{\mu} \neq 0 . Q(z)$ is a rational function, nonpositive on $|z|=1$, and this implies that $Q(0) \neq 0$ too. At $z=0, z f^{\prime}(z)=0$. Therefore, the quantity inside the square brackets of $\left(3^{\prime \prime}\right)$ must behave like $O\left(1 / z^{2}\right)$ around the origin. This implies that $f(z)$ has one finite simple pole at $z_{1}$, say and $f\left(1 / z_{1}\right)=f(0)$. So $f(z)=z+a_{0}+a_{1} /\left(z-z_{1}\right)$. The additional requirement that $f$ is a slit mapping shows that $f(z)=z+a_{0}+\left(z_{1}-1\right)^{2} /\left(z-z_{1}\right)$ with $z_{1}=\left(1+e^{i \theta}\right) / 2$. This is a circular arc mapping.

We remark that we have shown implicitly in the proof that the circular arc mapping is extremal for the functional $L(f)=f^{\prime}\left(z_{1}\right)$. This has been worked out by Grunsky and subsequently by Jenkins. See [6, Corollary 6.12] for references to this problem. It is unknown to us whether the circular arc mapping is extremal for another linear functional.

Proposition 1 can be slightly generalized to include functions with logarithmic singularities.

PROPOSITION 2. Let $f$ be an analytic slit mapping. If $f^{\prime}$ is rational, then $f$ has the form $z+a_{0}+a_{1} /\left(z-z_{1}\right)$.

Proof. Again the kernel $K(z)=\left(1 / z^{2}\right)\left(1-\bar{f}^{\prime}(1 / z)\right)$ is rational. So $w=f(z)$ satisfies the equation

$$
P(w)=\frac{1}{2 \pi} \int_{|\varsigma|=r} \frac{K(\varsigma)}{f(\varsigma)-w} d \varsigma=\frac{Q(z)}{z^{2} f^{\prime}(z)^{2}}
$$

with $P(w)$ rational in $w . Q(z)$ is rational in $z$. Thus $w=f(z)$ is an algebraic function. Since $f^{\prime}$ is rational, all the singularities of $f$ are poles. We are back to the case in Proposition 1.

\section{REFERENCES}

1. D. Aharonov, A note on slit mapping, Bull. Amer. Math. Soc. 75 (1969), 836-839.

2. A. Chang, M. Schiffer and G. Schober, On the second variation for univalent functions, J. Analyse Math. 40 (1981), 203-238. 
3. P. Duren, Univalent functions, Springer-Verlag, New York, 1983.

4. , Truncation, Contemporary Math. (to appear).

5. D. Hamilton, Extreme points of $\Sigma$, Proc. Amer. Math. Soc. 85 (1982), 393-396.

6. J. Jenkins, Univalent functions and conformal mappings, Springer-Verlag, Berlin, 1958.

7. R. Kühnau, Review of the article Support points with maximum radial angle, Math. Reviews $84 f$ (1984), 2204.

8. Y. Leung and G. Schober, Low order coefficient estimates in the class $\Sigma$, Ann. Acad. Sci. Fenn. Ser. A I Math. (to appear).

9. A. Pfluger, Lineare extremalprobleme bei schlichten funktionen, Ann. Acad. Sci. Fenn. Ser. A I Math. 489 (1971).

10. C. Pommerenke, Univalent functions, Vandenhoeck and Ruprecht, Göttingen, 1975.

11. S. Ruscheweyh, private communication.

12. G. Schober, Univalent functions-Selected topics, Lecture Notes in Math., volume 478, Springer-Verlag, Berlin and New York, 1975.

13. , Some conjectures for the class $\Sigma$, Contemporary Math. (to appear).

14. U. Srebro, Is the slit of rational slit mapping in $S$ straight? Proc. Amer. Math. Soc. 96 (1986), 65-66.

Department of Mathematics, University of Petroleum and Minerals, DHAHRAN, SAUdi ARABIA

Department of Mathematics, University of Delaware, NeWARk, Delaware 19716 